\title{
SIRT3 protects hepatocytes from oxidative injury by enhancing ROS scavenging and mitochondrial integrity
}

\author{
Jingxin Liu', Dan Li $i^{1}$, Tian Zhang ${ }^{1}$, Qiang Tong ${ }^{2}$, Richard Dequan Ye ${ }^{1}$ and Ligen Lin ${ }^{\star, 1}$
}

Evidences of oxidative stress and mitochondrial dysfunction have been recognized in most of clinical and experimental liver diseases. SIRT3, a member of NAD+-dependent deacetylases, is mainly localized in mitochondria. So far, the role of SIRT3 in protecting hepatocytes against oxidative stress remains elusive. Herein, we found SIRT3 protein expression is decreased in tertbutyl hydroperoxide ( $t$-BHP)-treated AML12 cells in vitro and primary hepatocytes from $\mathrm{CCl}_{4}$-injured mice in vivo. To further verify the role of SIRT3 in protecting hepatocytes from t-BHP-induced injury, SIRT3 overexpressed AML12 cell line and primary hepatocytes were generated. SIRT3 overexpressed hepatocytes showed improved cell viability upon $t$-BHP challenge, with less intracellular reactive oxygen species (ROS) accumulation. SIRT3 overexpression reduced superoxide dismutase 2 acetylation level and stimulated nuclear factor erythroid 2-related factor 2 nuclear translocation to enhance anti-oxidative capacity. Moreover, SIRT3 deacetylated peroxisome proliferator-activated receptor $\gamma$ coactivator $1 \alpha$ to promote mitochondrial biogenesis, and 8-oxoguanine DNA glycosylase 1 to orchestrate DNA repair, resulting in improved mitochondrial function. Through deacetylating Ku70, SIRT3 also abated mitochondrial translocation of dynamin-related protein 1, to attenuate mitochondrial fragmentation in $t$-BHP-injured hepatocytes. These results suggested that SIRT3 protected hepatocytes against oxidative stress by enhancing ROS scavenging and maintaining mitochondrial integrity.

Cell Death and Disease (2017) 8, e3158; doi:10.1038/cddis.2017.564; published online 26 October 2017

Evidence of oxidative stress has been recognized in most of clinical and experimental liver diseases, including alcoholic liver disease, hepatitis $\mathrm{C}$ virus infection, nonalcoholic fatty liver disease, and genetic hemochromatosis. ${ }^{1}$ During liver damage, reactive oxygen species (ROS) cause hepatocyte dysfunction and apoptosis, infiltration of Kupffer cells into the liver, and activation of hepatic stellate cells. ${ }^{2}$ ROS are mainly generated from the endoplasmic reticulum and the electron transport chain in the mitochondria of hepatocytes. Hepatocytes maintain balance between antioxidants and oxidants under normal conditions. Oxidative stress is a state when the cellular oxidant/antioxidant redox balance is altered in favor of the oxidant state. ${ }^{3}$ Thus, targeting excessive ROS accumulation is an effective method to attenuate oxidative stress-induced liver damage. Mitochondrial dysfunction is often associated with increased ROS production. ROS decrease mitochondrial function by affecting the replication and transcription of mitochondrial DNA, causing enhanced ROS production and further damage to mitochondrial DNA. ${ }^{2}$ Mitochondrial ROS homeostasis is important in preventing oxidative injury in hepatocytes.

Impaired mitochondria definitely link with the onset and perpetuation of liver diseases. Altered mitochondrial functions have indeed been documented in a variety of chronic liver diseases including hepatic steatosis, nonalcoholic fatty liver disease, and viral hepatitis. ${ }^{4,5}$ Dysfunction of mitochondria indicates loss of mitochondrial integrity including impairment of the electron transport chain, mitochondrial fragmentation, mitochondrial DNA damage. Adequate mitochondrial integrity maintains activity of critical enzymes. ${ }^{6}$

SIRT3, a member of $\mathrm{NAD}^{+}$-dependent deacetylases, is mainly localized in mitochondria. ${ }^{7}$ Lysine acetylation is a posttranslational modification that regulates mitochondrial enzyme activity, and $>20 \%$ of mitochondrial proteins are regulated by acetylation at lysine residues. ${ }^{8}$ The SIRT3 protein is transported into the mitochondrial matrix and then activated through proteolytic processing at the N-terminus. ${ }^{9,10}$ SIRT3 expression is increased in response to fasting, caloric restriction, or exercise. ${ }^{11,12}$ SIRT3 deacetylates many enzymes involved in response to oxidative stress and mitochondrial integrity. Peroxisome proliferator-activated receptor $\gamma$ coactivator $1 a(\mathrm{PGC}-1 a)$ regulates mitochondrial biogenesis, which is critical to the maintenance of energy metabolism and mitochondrial function. ${ }^{13}$ Recently, it was reported that SIRT3 upregulates the expression of PGC-1a through deacetylation of FOXO3. ${ }^{14}$ SIRT3 deacetylates superoxide dismutase 2 (SOD2) ${ }^{15}$ and isocitrate dehydrogenase $2^{16}$ to augment anti-oxidative capacity. Recent studies have disclosed that SIRT3 is involved in promoting mitochondrial biogenesis and correcting mitochondrial structure defects. ${ }^{14,17}$ Moreover, Ku70 is capable of binding with SIRT3 physically to be deacetylated, which results in impeding of Bax mitochondrial translocation. ${ }^{18,19}$ However, the role of SIRT3 in protecting hepatocytes against oxidative stress is not fully characterized.

\footnotetext{
${ }^{1}$ State Key Laboratory of Quality Research in Chinese Medicine, Institute of Chinese Medical Sciences, University of Macau, Avenida da Universidade, Macau, China and ${ }^{2}$ Children's Nutrition Research Center, Baylor College of Medicine, Houston, TX, USA

${ }^{*}$ Corresponding author: L Lin, State Key Laboratory of Quality Research in Chinese Medicine, Institute of Chinese Medical Sciences, University of Macau, Avenida da Universidade, Taipa, Macau 999078, China. Tel: +853 88228041; Fax: +853 28841358. E-mail: ligenl@umac.mo

Received 11.5.17; revised 17.8.17; accepted 25.9.17; Edited by A Finazzi-Agro
} 
In the present study, we demonstrated oxidative stress depletes SIRT3 expression in liver, and uncovered the role of SIRT3 in enhancing ROS-scavenging through deacetylation of SOD2, and mitochondrial integrity through deacetylation of PGC- $1 a$ and Ku70.

\section{Results}

SIRT3 expression is decreased in oxidative injured hepatocytes in vitro and in vivo. $\mathrm{CCl}_{4}$ is known to cause acute liver injury characterized with centrilobular necrosis. ${ }^{20}$ We determined whether SIRT3 expression is changed in primary hepatocytes from $\mathrm{CCl}_{4}$-injured mice. In the liver injured mice, aspartate transaminase (AST, Figure 1a) and alanine transaminase (ALT, Figure $1 \mathrm{~b}$ ) levels were significantly increased. The H\&E staining was performed to observe the change of liver architecture. More necrosis and inflammation hepatocytes were found in the liver specimens from $\mathrm{CCl}_{4}$-treated mice, compared with those of control mice (Figure 1c). As expected, the SIRT3 protein expression was suppressed in the primary hepatocytes from oxidative injured mice (Figure 1d). It suggested SIRT3 might have a key role in protecting hepatocytes from oxidative stress.

Consistently, in the murine hepatocyte AML12 cells, the SIRT3 protein expression was also decreased when treated with tert-butyl hydroperoxide ( $t$-BHP), a widely used oxidative injury inducer (Figure 2a). To directly evaluate the role of SIRT3 in hepatocytes against oxidative stress, we first generated an AML12 cell line expressing a transgene in which the Flag-tag fused to N-terminus of the full-length SIRT3 CDNA (SIRT3OV). ${ }^{21}$ SIRT3OV cells expressed around 3.5fold more SIRT3 protein than that in the cells transfected with vector plasmid (vector) using western blot analysis (Figure $2 \mathrm{~b}$ ). In addition, the Flag protein was only detected in the SIRT3OV cells (Figure 2b). Interestingly, SIRT3 overexpression exerted profound effect on total protein acetylation levels and apparently downregulated acetylation levels of most protein (Supplementary Figure S1a). Evidence of SIRT3 in protecting hepatocytes from $t$-BHP-induced oxidative injury was evaluated by 3-(4,5-dimethylthiazol-2-yl)-2,5-diphenyltetrazolium bromide (MTT) method. The viability of SIRT3OV cells was $\sim 30 \%$ higher than that of the vector cells under $500 \mu \mathrm{M} t$-BHP treatment, but unchanged under normal condition (Figure 2c), which was further supported by the trypan blue exclusion results (Supplementary Figure S1b). The above data indicated that overexpression SIRT3 significantly protected hepatocytes against oxidative injury.

\section{SIRT3 enhanced cellular anti-oxidative defense capacity.} Prevention of excessive ROS directly relies on antioxidants in cells. $^{22}$ Then, the anti-oxidative capacity of SIRT3OV cells was examined. Using 2,7-dichlorodihydrofluorescein diacetate (DCFH-DA) assay, the intracellular ROS content was significantly boosted in AML12 cells treated with $t$-BHP; and SIRT3 effectively reduced the ROS content in $t$-BHP-injured cells, but not in normal cells (Figure 3a). And the concentration of malondialdehyde, the lipid peroxide product, was also significantly decreased in SIRT3OV cells (Supplementary Figure S2a). GSH is an important non-enzymatic antioxidant, which is capable to prevent ROS-induced damage to important cellular components. ${ }^{23}$ As expected, the GSH level was significantly reduced in the $t$-BHP-treated AML12 cells, which was restored in SIRT3OV cells (Figure $3 b$ ). Nuclear factor erythroid 2-related factor 2 (Nrf2) is the master regulator of antioxidant responses, which controls GSH production and regeneration. ${ }^{24}$ Indeed, SIRT3 overexpression remarkably increased the total Nrf2 levels under normal and $t$-BHP-treated conditions (Figure 3c). NRF2 is bound and maintained in cytoplasm by its inhibitor KEAP1 (Kelch-like
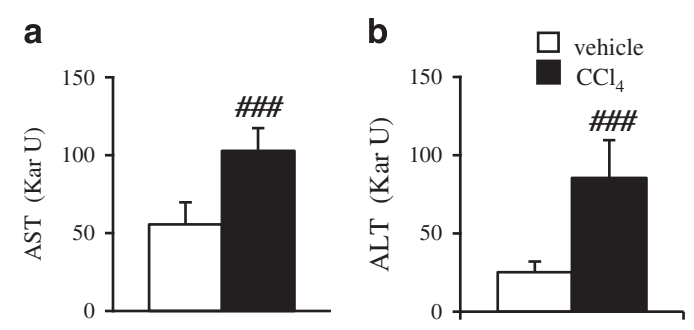

d
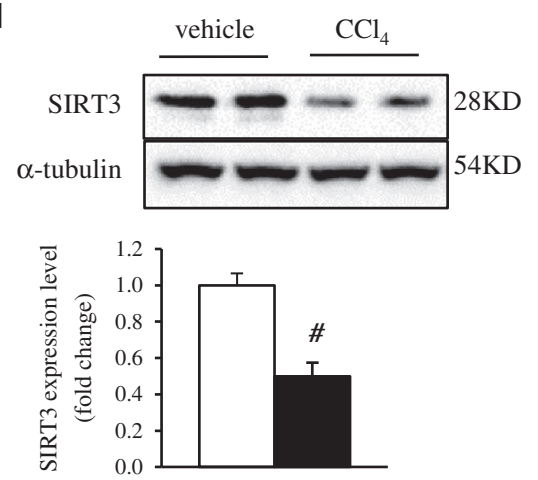

C
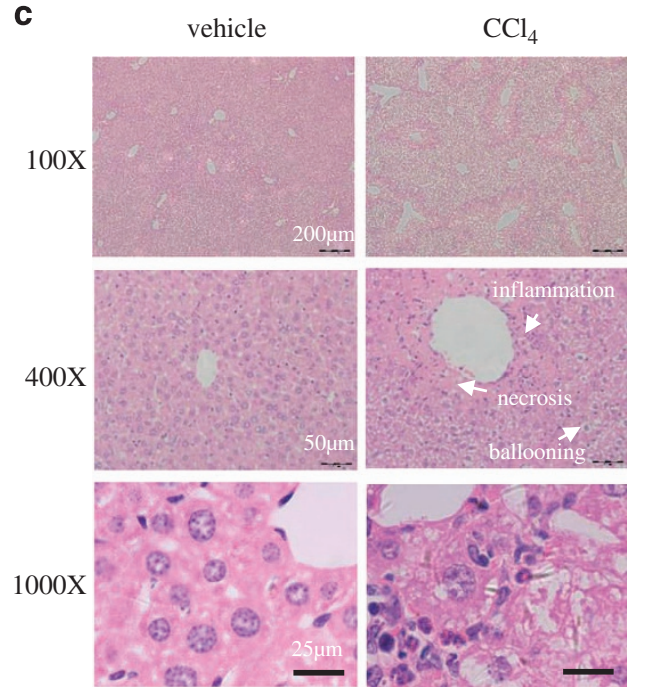

Figure 1 SIRT3 expression is decreased in primary hepatocytes from $\mathrm{CCl}_{4}$-induced hepatic damage mice. (a) Serum AST levels in mice. (b) Serum ALT levels in mice. (c) Representative H\&E staining of mouse livers. (d) SIRT3 protein levels in mouse livers. Data are shown as mean \pm S.D., $n=10-12,{ }^{\# \#} P<0.001 \mathrm{CCl}_{4}$ versus vehicle group 
a

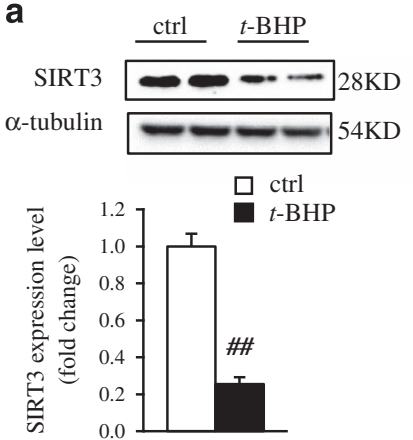

b

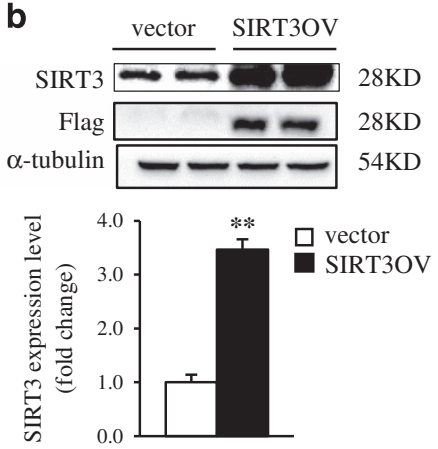

C

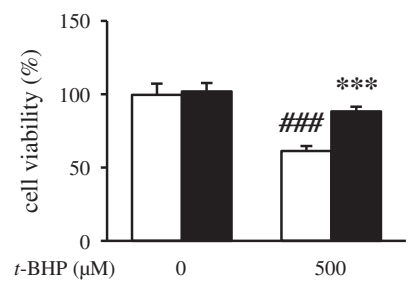

Figure 2 SIRT3 protects AML12 hepatocytes from t-BHP-induced oxidative injury. (a) SIRT3 protein is decreased in t-BHP-treated AML12 cells. (b) Generation of SIRT3 overexpressed AML12 cell line. SIRT3 protein levels were detected by western blot analysis and quantified using Image J. (c) Cell viability was determined by MTT assay. ${ }^{* \star} P<0.01$, and ${ }^{* \star *} P<0.001$ SIRTOV versus vector cells, and ${ }^{\# \#} P<0.01$ and ${ }^{\# \# \#} P<0.001 t$-BHP-treated versus ctrl cells

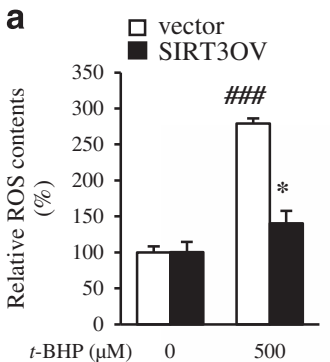

b

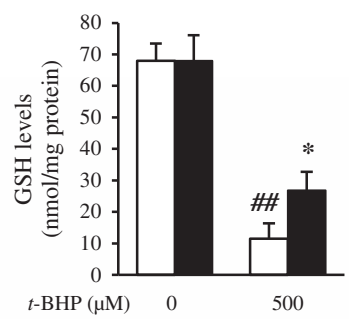

C

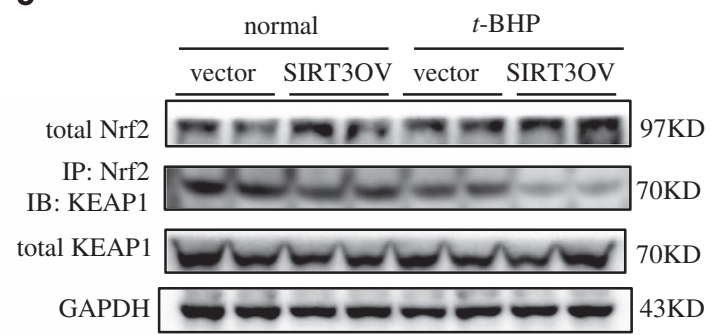

d

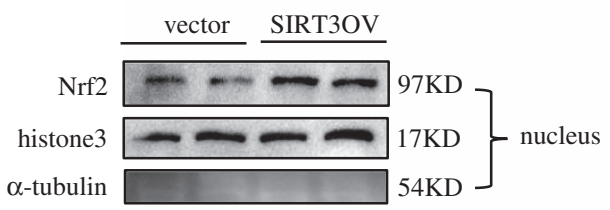

e

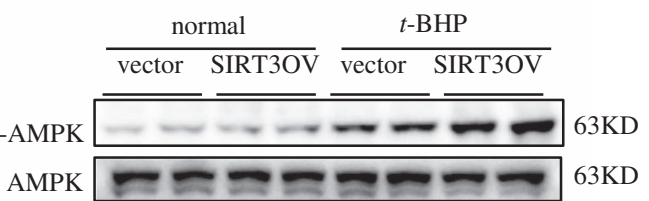

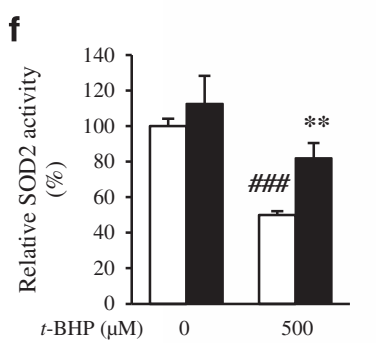

g

Ac-SOD2

SOD2

$\beta$-actin

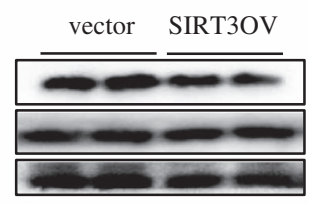

$25 \mathrm{KD}$ $\left.\begin{array}{l}25 \mathrm{KD} \\ 45 \mathrm{KD}\end{array}\right\}$ input

h $\frac{\text { normal }}{\text { vector SIRT3OV }} \frac{t \text {-BHP }}{\text { vector SIRT3OV }}$

catalase

GAPDH

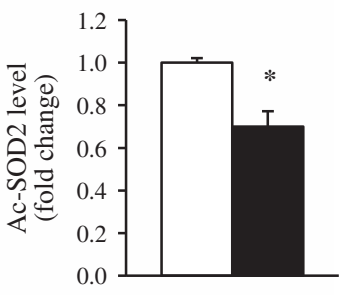

$64 \mathrm{KD}$ 43KD

Figure 3 SIRT3 overexpression enhances cellular anti-oxidative defense capacity. (a) Intracellular ROS level. (b) GSH level. (c) Total Nrf2 and Keap1 protein levels. (d) Nuclear Nrf2 protein level. (e) Phosphorylated and total AMPK protein levels. (f) SOD2 activity. (g) Acetylated and total SOD2 protein levels. (h) Catalase protein level. Data are shown as mean \pm S.D., $n=6,{ }^{*} P<0.05$ and ${ }^{* *} P<0.01$, vector versus SIRT3OV cells, ${ }^{\# \#} P<0.01$, and ${ }^{\# \# \# ~} P<0.001 t$-BHP-treated versus ctrl cells

$\mathrm{ECH}$-associated protein 1), which is released to regulate expression of anti-oxidative genes upon oxidative stress. ${ }^{25}$ Although the total protein did not change, the KEAP1 protein bound to Nrf2 was obviously reduced in the SIRT3OV cells compared with that in the vector cells, under both normal and t-BHP-treated conditions (Figure 3c). It suggested that SIRT3 might promote the release of KEAP1 from Nrf2 to stimulate Nrf2 nuclear translocation. Furthermore, the nuclear Nrf2 protein was increased in SIRT3OV cells (Figure 3d). The nuclear translocation of Nrf2 was further confirmed with the immunostaining images (Supplementary Figure S2b). SIRT3 overexpression also increased the phosphorylation level of adenosine 5'-monophosphate (AMP)-activated protein kinase (AMPK) (Figure 3e). SIRT3 was reported to directly deacetylate SOD2 and regulate its activity. ${ }^{15}$ As expected, SOD2 activity was reduced in $t$-BHP-treated AML12 cells, and SIRT3 overexpression markedly restored SOD2 activity (Figure 3f). Although the total SOD2 protein did not change, SIRT3 overexpression markedly reduced the acetylation level of SOD2 in AML12 cells (Figure $3 \mathrm{~g}$ ). Moreover, catalase 
expression was obviously increased in SIRT3OV cells, especially under $t$-BHP treatment (Figure $3 \mathrm{~h}$ ). These results indicated that SIRT3 enhances ROS-scavenging capability in oxidative injured hepatocytes.

SIRT3 reversed $t$-BHP-induced loss of mitochondria in hepatocytes. ROS damage is associated with a decline in mitochondrial integrity featured with damage of mitochondrial DNA and proteins. ${ }^{26}$ The Mitotracker Green staining results showed SIRT3 overexpression significantly increased the mitochondrial content in the $t$-BHP-treated hepatocytes (Figure 4a). Furthermore, the flow cytometry results revealed a $50 \%$ increase of mitochondrial mass in SIRT3OV cells (Figure 4b), which was further supported by the higher ratio of mtDNA/nuclear DNA in SIRT3OV hepatocytes compared with that in the vector cells (Figure 4c). PGC-1a is the master regulator of mitochondrial biogenesis, and its activity is negatively associated with the acetylation level. ${ }^{27}$ Interestingly, SIRT3 overexpression not only upregulated PGC-1a protein but also reduced its acetylation level (Figures $4 d$ and e). In addition, the mitochondrial structure proteins, Sam50 and Tom40, were also increased in SIRT3 overexpressed hepatocytes, especially under $t$-BHP treatment (Figure $4 d$ ). Decreased acetylation of $\mathrm{PGC}-1 a$ resulted in the nuclear translocation, characterized by the immunostaining images (Figure 4f). Consistently, the mitochondrial transcription factor $A$, a key participant in mitochondrial genome replication, was significantly increased in SIRT3OV cells (Supplementary Figure S3a). These results suggested that SIRT3 promotes mitochondrial biogenesis in hepatocytes under oxidative stress.

8-Oxoguanine (8-oxo-dG), an oxidized form of guanine, is a marker of DNA oxidative damage. Our result showed 8-oxodG level was lower in SIRT3OV cells than that in the vector cells (Figure 4g). 8-Oxoguanine DNA glycosylase (OGG1) is a DNA repair enzyme serving as a deacetylation substrate of SIRT3 in cancer cells. ${ }^{28}$ Western blot analysis showed SIRT3 overexpression increased total OGG1 protein and decreased its acetylation level in oxidative injured hepatocytes (Figure $4 \mathrm{~h}$ and Supplementary Figure S3b). Mitochondrial membrane potential $(\Delta \Psi \mathrm{m})$ plays a vital role in maintaining the physiological function of mitochondria. ${ }^{29} \mathrm{R} 123$ was used as a mitochondrial membrane potential probe because it quenches when entering the mitochondrial membrane in a membrane potential-dependent manner. The results showed $t$-BHP treatment interrupted $\Delta \Psi \mathrm{m}$; SIRT3OV cells possessed higher $\Delta \Psi \mathrm{m}$, indicating improved mitochondrial function (Figure $4 \mathrm{i}$ ). Taken together, these results suggested that SIRT3 overexpression enhanced mitochondrial content and function in hepatocyte under oxidative stress.

SIRT3 attenuated mitochondrial fragmentation in oxidative injured hepatocytes. Mitochondrial morphology is a result of its dynamic changes and abnormal mitochondrial morphology is associated with a variety of disorders and dysfunctions. The balance of fusion and fission events plays a pivotal role in maintaining mitochondrial architecture and function. In the current study, the severe mitochondrial fragmentation was observed in $t$-BHP damaged hepatocytes and SIRT3 overexpression ameliorated the mitochondrial fragmentation (Figure 5a). Drp1 (dynamin-related protein 1) is a conserved GTPase protein that mediates membrane remodeling in mitochondria. Drp1 resides mainly in the cytoplasm with only a small fraction localizing to mitochondria as foci, which is involved in mitochondrial fission. ${ }^{30}$ When activated, Drp1 was recruited to mitochondrial fission sites to process fission which is essential for maintaining mitochondrial number and function. On the contrary, Drp1would lead mitochondrial fragmentation under imbalance of fusion and fission. Thus, we speculated that SIRT3 abated mitochondrial fragmentation mediating through Drp1 recruitment to mitochondria. The immunostaining images showed SIRT3 overexpression significantly inhibited Drp1 mitochondrial recruitment in the $t$-BHP-treated hepatocytes (Figure $5 b$ ). Consistently, the western blot analysis showed the mitochondrial Drp1 protein level was reduced in SIRT3OV cells under $t$-BHP treatment, but not in normal cells (Figure $5 \mathrm{c}$ ). Mff (mitochondrial fission factor) and MiD49 (mitochondrial dynamics protein of $49 \mathrm{KDa}$ ) mediate Drp1 mitochondrial translocation. ${ }^{31}$ The results showed the protein expressions of Mff and MiD49 were decreased in SIRT3OV hepatocytes with or without treatment of oxidant (Figure 5c). It was reported that cytosolic Bax targets the mitochondrial outer membrane and co-localizes with Drp $1 ;{ }^{32}$ and Bax is essential for Drp1-mediated mitochondrial fission. ${ }^{33} \mathrm{Ku} 70$, one of the SIRT3 substrates, has capability of hindering the translocation of Bax to mitochondria. ${ }^{19}$ As expected, SIRT3 overexpression dramatically decreased Bax protein in mitochondria from $t$-BHP injured AML12 cells, but not from normal cells (Figure $5 \mathrm{c}$ ). Although the total Ku70 expression did not change, the acetylated Ku70 was significantly decreased in SIRT3OV hepatocytes (Figure $5 d$ ). The above data indicated SIRT3 attenuates mitochondrial fragmentation in oxidative injured hepatocytes mediating through Ku70-BaxDrp1 axis.

SIRT3 protects primary hepatocytes from oxidative injury. To confirm the protecting role of SIRT3, we isolated primary hepatocytes from liver of C57BL6 mice, and overexpressed SIRT3 in the primary hepatocytes (SIRT3OVprimary). As shown in Figure 6a, the SIRT3 protein was 2.3fold higher in SIRT3OV-primary cells compared with that in the primary hepatocytes expressed vector control (vectorprimary). Consistently, SIRT3 overexpression in primary hepatocytes stimulated Nrf2 nuclear translocation (Figure 6b), increased catalase level and reduced SOD2 acetylation level (Figure 6c), to enhance anti-oxidative capacity. Moreover, SIRT3 overexpression activated AMPK and reduced PGC-1 $a$ acetylation level (Figure $6 \mathrm{~d}$ ), to regulate mitochondrial biogenesis. At last, SIRT3 overexpression decreased Drp1 and Bax proteins in mitochondria, and reduced acetylated Ku70 level, to attenuate mitochondrial fragmentation (Figure $6 \mathrm{e}$ ). In addition, the primary hepatocytes from $\mathrm{CCl}_{4}$-injured mice showed less cell viability (Figure 6f), more intracellular ROS accumulation (Figure $6 \mathrm{~g}$ ) and reduced mitochondrial membrane potential (Figure 6h), when compared with those from control mice. Taken together, overexpression of SIRT3 protects primary hepatocytes from oxidative injury. 
a

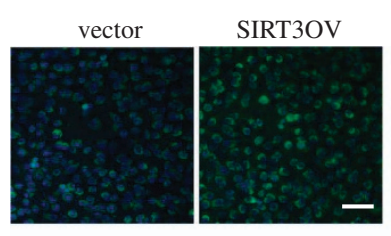

b

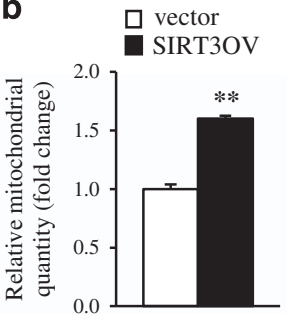

C

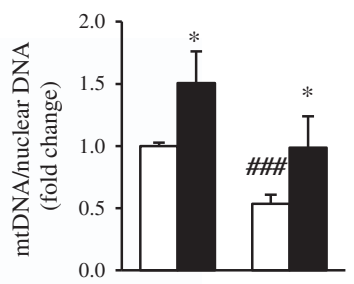

d $\frac{\text { normal }}{\text { vector SIRT3OV }} \frac{t \text {-BHP }}{\text { vector SIRT3OV }}$

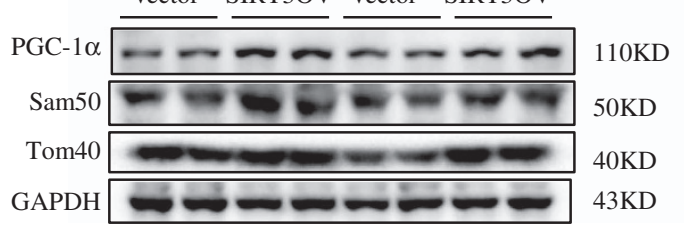

e
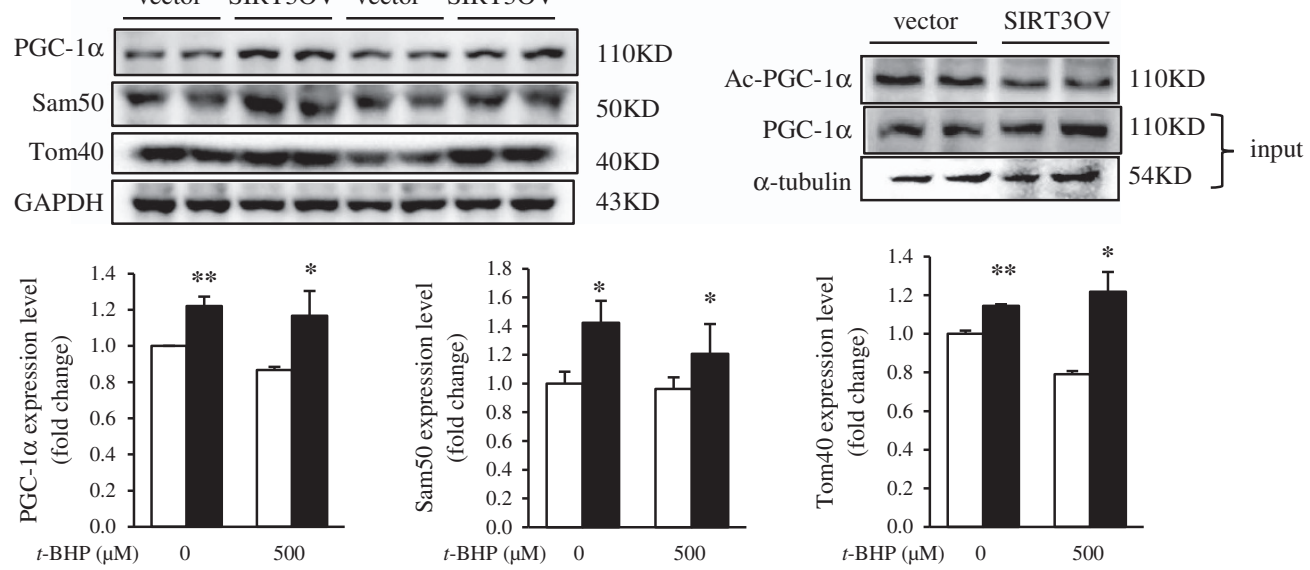

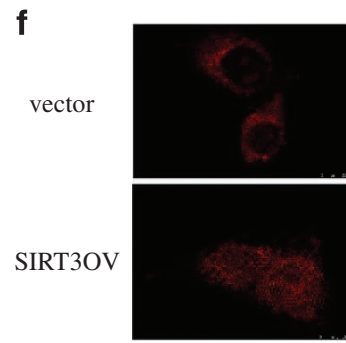

PGC-1 $\alpha$

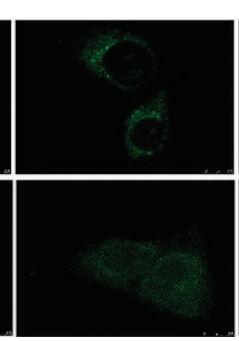

Mitotracker

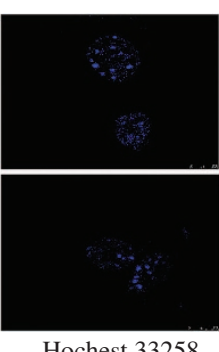

Hochest 33258

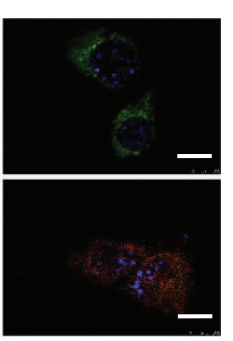

merge
9

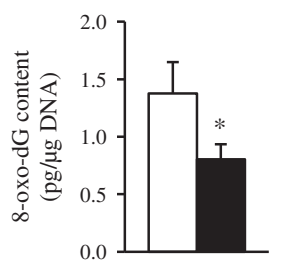

i

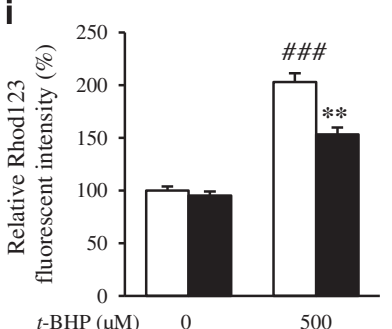

Figure 4 SIRT3 prevents t-BHP-induced mitochondrial loss in AML12 hepatocytes. (a) Mitochondria were visualized using Mitotracker Green (Green) and nuclei were visualized using DAPI (blue) in an IN Cell Analyzer. Scale bar $100 \mu \mathrm{m}$. (b) Quantification of mitochondrial quantity by flow cytometry. (c) Ratio of mitochondrial DNA/nuclear DNA. (d) Protein expressions of PGC-1 $\alpha$, Sam50, and Tom 40 in vector and SIRT3OV cells, under both normal and induction conditions. (e) Acetylated and total PGC-1 $\alpha$ protein levels. (f) Immunofluorescence analyses of PGC-1 $\alpha$ levels. Cellular PGC-1 $\alpha$ levels were observed using a Leica TCS SP8 Confocal Laser Scanning Microscope System. Cells were stained with Mitotracker Green (green), PGC-1 $\alpha$ (red), and Hoechst 33258 (blue). Scale bar, $10 \mu \mathrm{m}$. (g) Change in 8-oxo-dg level in vector and SIRT30V cells treated with $500 \mu \mathrm{M}$ t-BHP. (h) Acetylated and total OGG1 protein levels. (i) Relative Rhod123 fluorescent intensity in vector and SIRT3OV cells, under both normal and induction conditions. Data are shown as mean \pm S.D., $n=6,{ }^{*} P<0.0,5$ and ${ }^{* *} P<0.01$ vector versus SIRT3OV cells, ${ }^{\# \# \#} P<0.001 t$-BHP-treated versus ctrl cells

SIRT3 silenced deteriorated oxidative injury in hepatocytes. To further verify the role of SIRT3 in protecting hepatocytes from oxidative injury, a SIRT3-silenced AML12 cell line (SIRT3 silenced) was generated using shRNAtargeting SIRT3. As shown in Figure 7a, SIRT3-silenced cells expressed $\sim 60 \%$ less SIRT3 protein compared with that of the cells expressing scrambled shRNA (scrambled). Treatment of $t$-BHP caused more severe cell death in SIRT3silenced cells compared with that of scrambled cells (Figure 7b). As expected, SIRT3 silenced decreased 

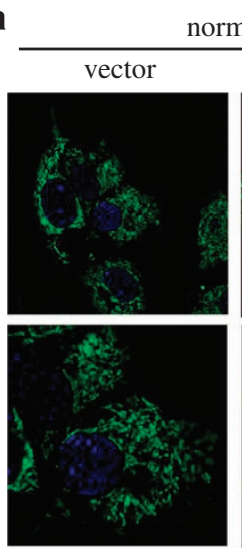

normal
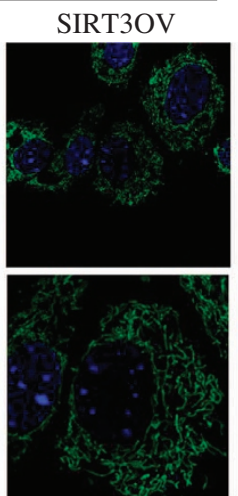
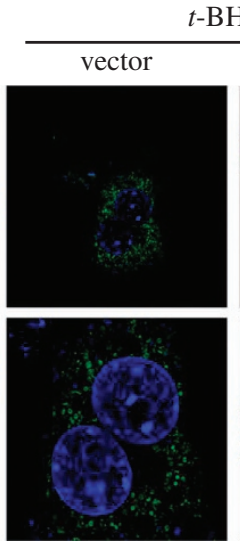

$t$-BHP
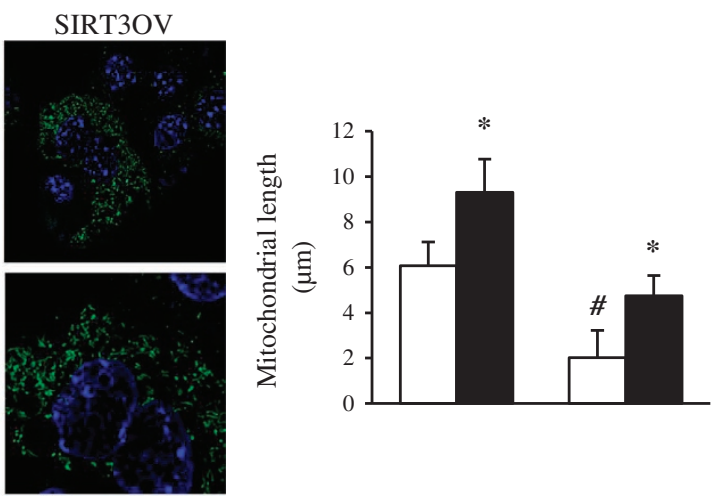

b

DAPI

Drp1

Mitotracker

merge

colocalization

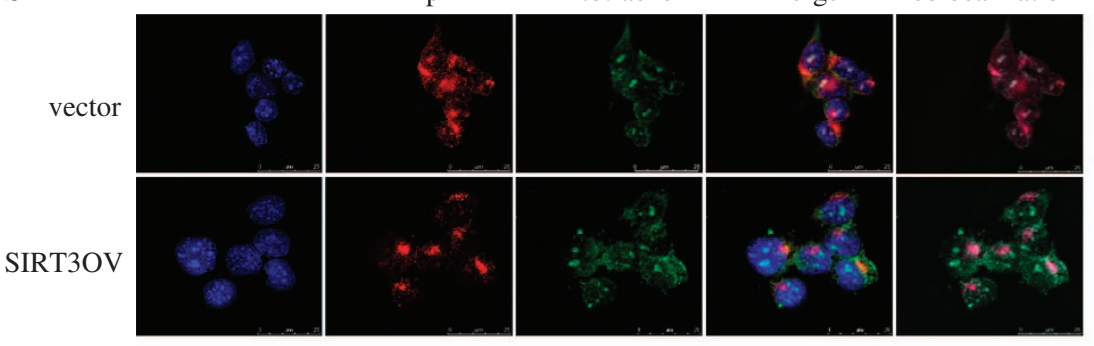

C

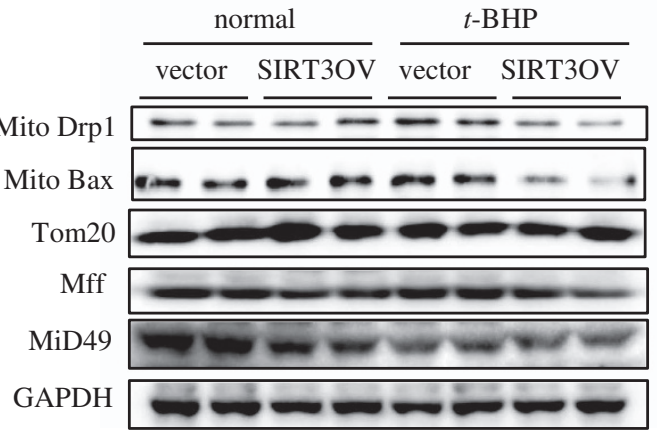

d

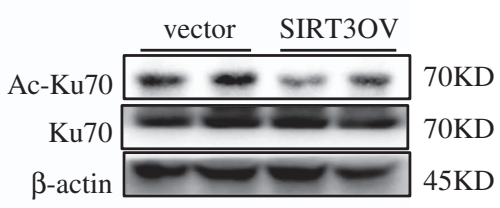

$16 \mathrm{KD}$

$37 \mathrm{KD}$

$49 \mathrm{KD}$

$43 \mathrm{KD}$

$78 \mathrm{KD}$

$21 \mathrm{KD}$

Figure 5 SIRT3 prevents $t$-BHP-induced mitochondria fragmentation in AML12 hepatocytes. (a) Representative confocal image of mitochondrial morphology. Nuclei were visualized using Hochest 33258 (blue), and mitochondria were visualized using Mitotracker Green (green). Mitochondrial length was analyzed using Imaris7.4.2 software. After the indicated treatments, mitochondrial were stained with Mitotracker Green in hepatocytes. Z-stack images were analyzed for changes in mitochondrial structure using Imaris7.4.2 software, and 3D reconstructions were generated from z-stack images for analysis of mitochondrial length. (b) Immunofluorescence analyses of Drp1 localization. Cells were stained with Mitotracker Green (green), Drp1 (red), and DAPI (blue). Scale bar, $25 \mu \mathrm{m}$. (c) Drp1 and Bax protein levels in mitochondrial compartment, and Mff and Dip49 protein levels in whole cell lysate. (d) Acetylated and total Ku70 protein levels. Data are shown as mean \pm S.D., $n=6$, ${ }^{\star} P<0.05$ vector versus SIRT3OV cells, ${ }^{\sharp} P<0.05 t-$ BHP-treated versus ctrl cells

mitochondrial content, indicating by the flow cytometry analysis of Mitotracker Green (Figure 7c) and the ratio of mtDNA/nuclear DNA (Figure 7d). Consistently, the immunostaining of Tom20 showed a remarkable decrease of mitochondrial quantity in SIRT3-silenced cells (Figure 7e). On the other hand, SIRT3 silenced deteriorated mitochondrial fragmentation in hepatocytes under oxidative stress (Figure 7f). Specifically, the Mander's coefficient in SIRT3silenced cells was about threefold higher than that in the scrambled cells using threshold (3.04), suggesting SIRT3 plays an important role in Drp1 mitochondrial translocation. The western blotting results also showed that Drp1 and Bax protein levels were increased markedly in mitochondria from SIRT3-silenced cells (Figure $7 \mathrm{~g}$ ). In addition, the total Bax protein was also increased in SIRT3-silenced cells
(Figure 7g). Although the total Ku70 protein didn't change, the acetylation level of Ku70 was increased remarkably in SIRT3silenced cells (Figure 7h). Taken together, loss of SIRT3 causes decreased mitochondrial content and severe mitochondrial fragmentation in oxidative injured hepatocytes.

\section{Discussion}

SIRT3 has a vital role in manipulating oxidative stress in various cells and tissues. A previous study showed that SIRT3 expression in hepatocytes decreased in high-fat diet-induced obese mice. ${ }^{34} \mathrm{~A}$ recent study has suggested that SIRT3 protects oxidative stress mediated by hepatitis $B$ virus $X$ protein expression. ${ }^{35}$ The present results showed that SIRT3 expression is decreased in $t$-BHP-injured hepatocytes in vitro 
and primary hepatocytes from $\mathrm{CCl}_{4}$-induced liver damaged mice in vivo. Hence, oxidative injury affected SIRT3 expression in hepatocytes, indicating that SIRT3 has a key role in protecting oxidative stress in liver.

Liver contains a large number of mitochondria, which are the predominant source of intracellular ROS. Excessive ROS accumulation results in cell death through the oxidation of polyunsaturated fatty acids in cellular membranes, the substantial number of unprotected protein sulfhydryl groups, and DNA bases. ${ }^{36}$ In hepatocytes, the anti-oxidative system maintains a balance of ROS content, including SOD2, GSH, and catalase. SIRT3 directly deacetylates lysine 122 to regulate SOD2 activity in response to stress. ${ }^{37}$ The current data uncovered SIRT3 decreased the acetylation level of SOD2, but did not change the total protein level, to enhance SOD2 activity, in oxidative injured hepatocytes. Many studies revealed that adequate GSH level offers cellular protection against the noxious effect of intracellular ROS. ${ }^{38}$ The current data showed that SIRT3 overexpression increased GSH level, suggesting improved capacity to attenuate ROS-induced hepatic damage. Nrf2 is a pivotal transcriptional factor capable of recognizing the antioxidant response element through nuclear translocations. ${ }^{39}$ Interestingly, we observed SIRT3 overexpression released the inhibitor KEAP1 from Nrf2 and stimulates Nrf2 nuclear translocation. It is also notable that in some cases Nrf2 induces SIRT3 expression. ${ }^{40}$ Hence, the nuclear translocation of Nrf2 is capable of in turn strengthening SIRT3 function.
Mitochondria integrity is maintained through a series of physical processes such as mitochondrial biogenesis, autophagic of dysfunctional parts, and dynamics control on fission/ fusion events. In the present study, the mitochondrial homeostasis was significantly interrupted in the hepatocytes exposed to $t$-BHP, accompanied with decreased mitochondrial content and increased mitochondrial fragmentation. Interestingly, SIRT3 totally reserved mitochondrial integrity in hepatocytes under oxidative stress. In fact, mitochondrial biogenesis acts as a critical aspect for mitochondrial quantity, and SIRT3 was reported to facilitate the process by targeting PGC-1a deacetylation. ${ }^{27,41,42}$ The current data further supported SIRT3 promoted mitochondrial biogenesis through deacetylating PGC-1a in hepatocytes.

AMPK is responsible for monitoring cellular energy status and is regulated through phosphorylation at threonine $172 .{ }^{43}$ AMPK directly stimulates mitochondrial energy production and strengthens mitochondrial biogenesis. AMPK induces PGC-1a gene expression and directly phosphorylates PGC-1 $a^{44,45}$ In addition, liver kinase B1, an acetylation substrate of SIRT3, directly phosphorylates and activates AMPK. ${ }^{12,46}$ The current data showed that SIRT3 overexpression promoted the phosphorylation of AMPK, which could induce PGC-1a gene expression, and directly decrease the phosphorylation of PGC-1a. Phosphorylation of AMPK is also an underlying factor for Nrf2 nuclear translocation. ${ }^{47}$ In general, SIRT3 stimulates mitochondrial biogenesis in a

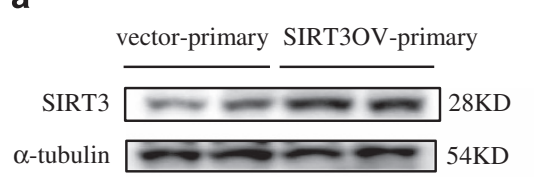

C

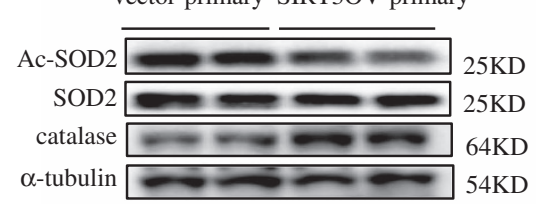

b

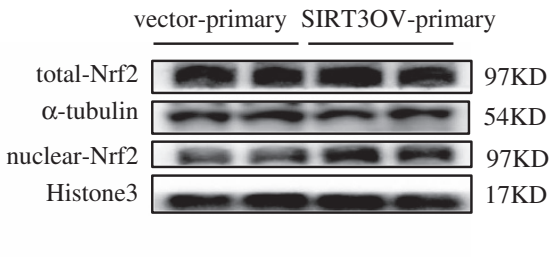

d

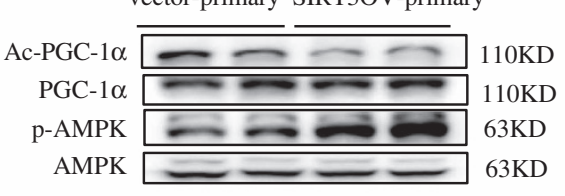

e

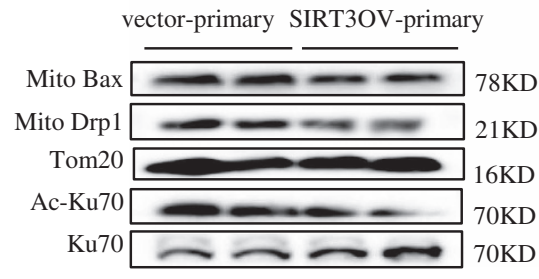

$\square$ vector-primary

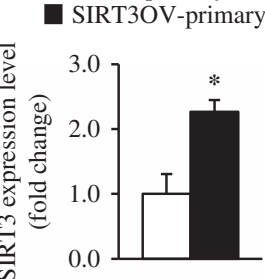


a

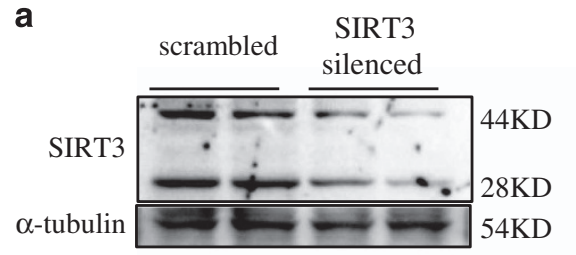

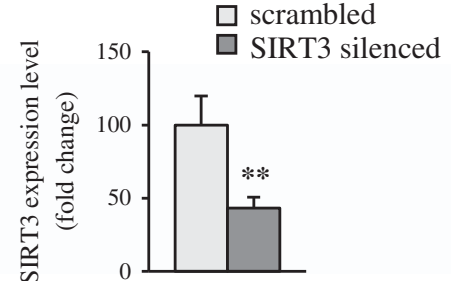

b

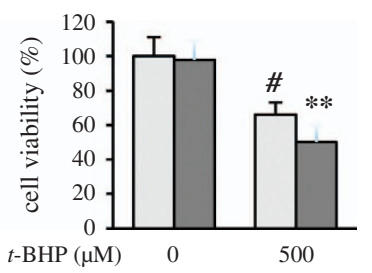

C

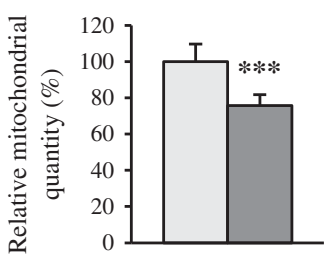

d

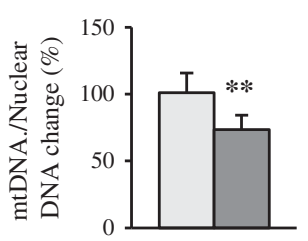

e

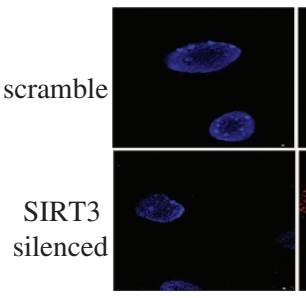

TOM20

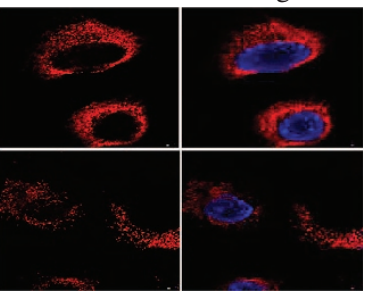

f

DAPI

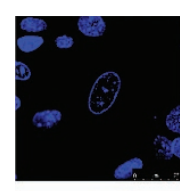

scrambled
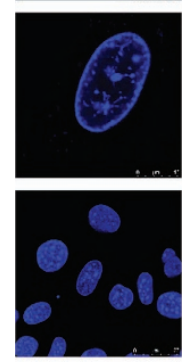

SIRT3

silenced
Mitotracker
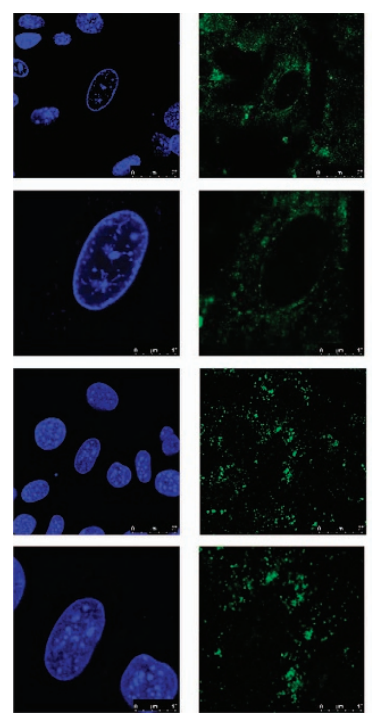

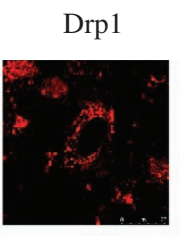

merge
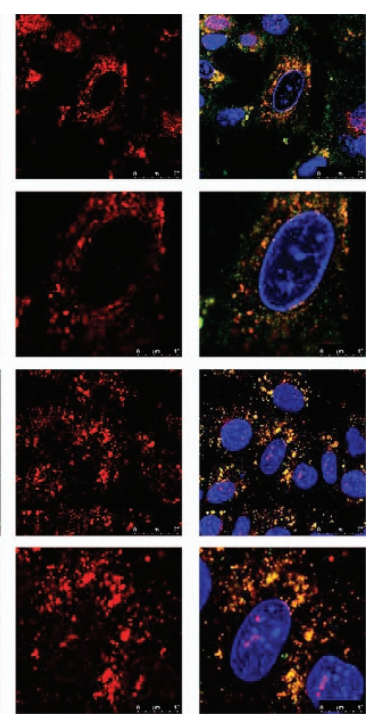

g

SIRT3

silenced

scrambled

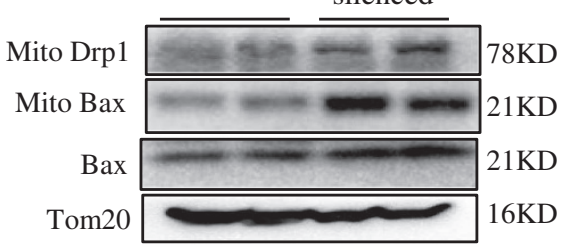

h
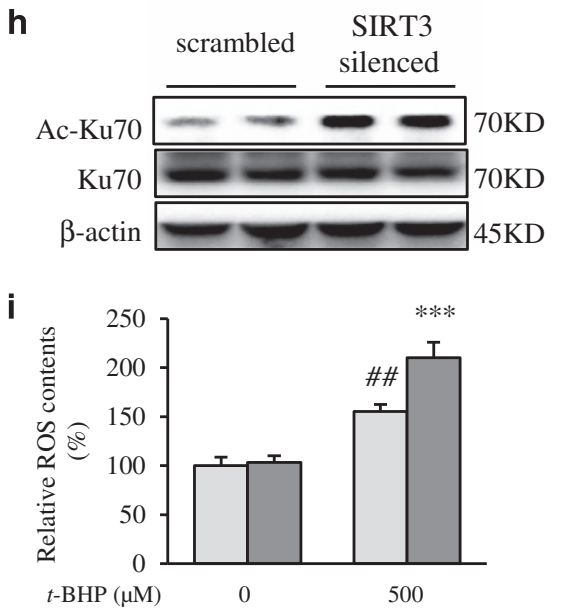

Figure 7 SIRT3 silenced deteriorated oxidative injury in hepatocytes. (a) Generation of SIRT3-silenced AML12 cell line expressing shScr or shSIRT3. SIRT3 protein levels in these cells were detected by western blot analysis and quantified using Image J. (b) Cell viability was determined by MTT assay before and after treatment of $500 \mu \mathrm{M} t$-BHP. (c) Quantification of mitochondrial content by Mitotracker Green. (d) Ratio of mitochondrial DNA/nuclear DNA. (e) Representative confocal image of mitochondrial morphology. Nuclei were visualized using Hochest 33258 (blue), and mitochondria were visualized using Tom20 (red). (f) Immunofluorescence analyses of Drp1 localization. Cells were stained with Mitotracker Green (green), Drp1 (red), and DAPI (blue). Scale bar, $25 \mu \mathrm{m}$. (g) Drp1 and Bax protein levels in mitochondrial compartment, and total Bax protein level. (h) Acetylated and total Ku70 protein levels. (i) Intracellular ROS level. ${ }^{* \star} P<0.01$ and ${ }^{\star \star *} P<0.001$ SIRT3-silenced versus scrambled cells, ${ }^{\#} P<0.05$ and ${ }^{\# \#} P<0.01 t$-BHP-treated versus ctrl cells

hepatocytes under oxidative stress through the SIRT3-AMPKPGC-1 $a$ axis.

SIRT3 may also involve in mitochondrial renewal and hepatocytes proliferation mediating through mitophagy mechanisms. Our preliminary data showed SIRT3 induced autophagy, leading to attenuation of dysfunctional mitochondria mediated cell injury (data not shown). Future studies are required to fully elucidate how SIRT3 regulates mitophagy when responding to oxidative stress.
Mitochondrial integrity, including DNA, protein, and organelle integrity, is important to maintain mitochondrial function. Mitochondria change their function, morphology, and even quantity in response to physiological conditions and stress environments, to keep integrity. ${ }^{48} 8-O x o-d G$ is a prevalent product of oxidative attacked DNA. ${ }^{49}$ 8-Oxo-dG can be fixed by the base excision repair pathway initiated by OGG1. Livers from OGG1 deletion mice showed up to sixfold higher levels of 8-oxo-dG in nuclear DNA and 20-fold higher in mitochondrial DNA compared with 


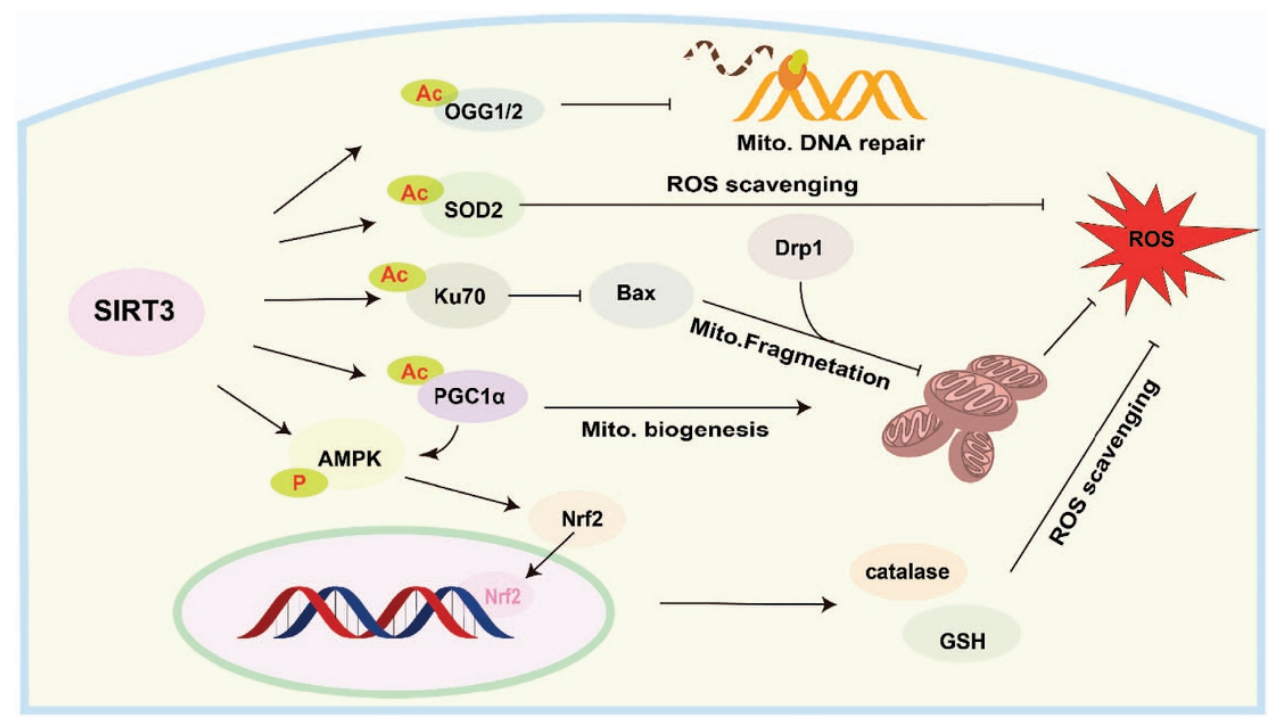

Figure 8 Schematic diagram of the role of SIRT3 in protecting hepatocytes from oxidative injury

those from wild-type mice. ${ }^{50}$ SIRT3 directly deacetylates OGG1 to protect mitochondrial integrity and prevent apoptotic cell death. ${ }^{51}$ The current data showed that SIRT3 overexpression decreased the acetylation level of OGG1 to reduce 8-oxo-dG content in the $t$-BHP-treated hepatocytes. It suggests SIRT3 has a critical role in repairing mitochondrial DNA damage to protect mitochondrial integrity under oxidative stress.

Mitochondria are highly dynamic organelles, which undergo continuous cycles of fusion and fission to guarantee cellular function. The balance between these two antagonistic processes helps to regulate mitochondrial morphology and maintain mitochondrial network integrity. Alterations of these two opposite processes are observed under both physiologic and pathological conditions. Fission contributes to control mitochondrial quantity and also alleviate the cellular load of damaged mitochondria. Unbalanced fission results in mitochondrial fragmentation. Drp1 is one of the principal proteins participating in mitochondrial fission. ${ }^{52}$ Overexpression of Drp1 promotes increased mitochondrial fission. ${ }^{53}$ We observed mitochondria suffered fragmentation in AML12 hepatocytes exposed to $t$-BHP-induced oxidative stress and SIRT3 overexpression alleviated it. Moreover, SIRT3 overexpression hindered Drp1 mitochondrial translocation through Ku70-Bax-Drp1 axis. Although SIRT3 might be involved in yet unknown fission mechanisms, our results supported that SIRT3 mitigates mitochondrial fragmentation, leading to attenuation of mitochondrial dysfunction and subsequent cell injury.

In conclusion, we determined SIRT3 level is decreased in oxidative injured hepatocytes in vitro and in vivo. More importantly, we verified SIRT3 protects hepatocytes against oxidative injury through enhancing anti-oxidative capacity, improving mitochondrial biogenesis, and preventing mitochondrial fragmentation, to maintain mitochondrial morphology and integrity (Figure 8). SIRT3 could be a potential target in the management of hepatic diseases.

\section{Materials and Methods}

Animals. Male C57Bᄂ/6 mice (aged 6-8 weeks) were purchased from the animal facility of Faculty of Health Sciences, University of Macau. The mice were fed a regular chow diet (Guangdong Medical Lab Animal Center, Guangzhou, Guangdong,
China) and water ad libitum. The mice were housed under standard light (i.e., $12 / 12 \mathrm{~h}$ light/dark), temperature $\left(21 \pm 2{ }^{\circ} \mathrm{C}\right)$, and relative humidity $(60 \pm 10 \%)$ conditions. The mice were randomly divided into two groups ( $n=10-12$ in each group). Acute liver damage was induced by oral administration with $158 \mathrm{mg} / \mathrm{kg} \mathrm{CCl}_{4}(1 \%$ (v/v) in olive oil) for the $\mathrm{CCl}_{4}$ groups of mice. Control group mice were treated with $10 \mathrm{~m} / \mathrm{kg}$ olive oil. After $24 \mathrm{~h}$, the mice were sacrificed under isoflurane anesthesia. Serum was obtained from blood via centrifugation at $3000 \times g$ for $20 \mathrm{~min}$. Hepatic samples of the middle of right lobe were fixed in $4 \%$ paraformaldehyde immediately for morphological examination, and the remaining liver tissues were snap frozen and stored at $-80^{\circ} \mathrm{C}$ for other studies. All animal experiments were approved by the Experimental Animal Ethics Committee at the University of Macau.

Determination of GSH, SOD2, AST, and ALT levels. The levels of GSH and SOD2 in AML12 cells and mouse livers, as well as the levels of AST and ALT in mouse serum, were determined using commercial assay kits (Nanjing Jiancheng, Nanjing, Jiangsu, China) in accordance with the manufacturer's protocols. Protein concentration was determined by a Pierce BCA Protein Assay Kit (Thermo Fisher, Rockford, IL, USA). GSH and SOD2 levels were normalized by total protein.

Cell culture. The murine hepatocyte AML12 cell line was purchased from American Type Culture Collection (Rockville, MD, USA). AML12 cells were cultured in DMEM supplemented with $10 \%$ FBS and ITS-G $(5 \mathrm{mg} / \mathrm{ml}$ insulin, $5 \mathrm{mg} / \mathrm{l}$ transferrin, and $5 \mu \mathrm{g} / \mathrm{l}$ selenous acid) (Peiyuan, Shanghai, China) in humidified air containing $5 \% \mathrm{CO}_{2}$ at $37^{\circ} \mathrm{C}$.

Generation of SIRT3 overexpression cell line. The pcDNA3.1-SIRT3flag plasmid was generated as previously described. ${ }^{54}$ The pcDNA3.1-SIRT3-flag and pcDNA3.1 plasmids were transfected into AML12 cells or primary hepatocytes using Lipofectamine 2000 (Invitrogen, Carlsbad, CA, USA) in accordance with the manufacturer's instruction ${ }^{55}$ In brief, AML12 cells $\left(4 \times 10^{5}\right)$ were seeded in $35 \mathrm{~mm}$ plates. After $24 \mathrm{~h}$, the cells were transfected with $10 \mu \mathrm{g}$ plasmids (pcDNA3.1 or pcDNA3.1-SIRT3-flag) using Lipofectamine 2000 reagent. At $24 \mathrm{~h}$ after transfection, $800 \mu \mathrm{g} / \mathrm{ml}$ G418 (Sigma, Santa Clara, CA, USA) was added to select positive cells for 12 days. The medium was changed every other day.

Cell viability assays. The viability of AML12 cells was determined using MTT. In brief, AML12 cells $\left(1 \times 10^{4}\right.$ cells/well) were seeded into a 96-well plate and then cultured for $16 \mathrm{~h}$. For vector and SIRT3OV cells, $500 \mu \mathrm{M} t$-BHP or the same volume of DMSO was added. After $12 \mathrm{~h}, 1 \mathrm{mg} / \mathrm{ml}$ MTT solution was added to each well, and the 96 -well plates were further incubated for $4 \mathrm{~h}$ at $37^{\circ} \mathrm{C}$. Subsequently, $100 \mu \mathrm{l}$ DMSO was added to each well to solubilize formazan precipitates. Absorbance at $570 \mathrm{~nm}$ was measured using a microplate reader (Flexstation 3, Molecular Devices, CA, USA). 
The cell viability was also evaluated by a trypan blue exclusion assay. Cells were seeded at a density of $5 \times 10^{4}$ per well in 12-well plates. The number of viable cells were counted by $0.4 \%$ trypan blue (Invitrogen) exclusion using a hemocytometer and expressed as a percentage relative to vector control.

Intracellular ROS detection. Intracellular ROS levels were detected using DCFH-DA (Invitrogen) as previously described. ${ }^{56}$ In brief, AML12 cells $\left(1 \times 10^{5}\right)$ were seeded into each well of a 96-well black multitier plate with a clear bottom and then cultured overnight. The cells were treated as described in cell viability section. After $12 \mathrm{~h}$, the cells were incubated with DCFH-DA $(10 \mu \mathrm{M})$ at $37^{\circ} \mathrm{C}$ in the dark for $15 \mathrm{~min}$. Fluorescence intensity was analyzed through FACSCalibur flow cytometry (BD, Lake Franklin, NJ, USA).

Immunoblotting. Primary hepatocytes and AML12 cells were lysed with RIPA lysis buffer (Beyotime, Shanghai, China) containing $1 \%$ protease inhibitor cocktail and $1 \%$ phenylmethane sulfonylfluoride (Sigma). Protein concentration was determined using a BCA Protein Assay Kit. An equal amount of proteins was separated using $5-12 \%$ sodium dodecyl sulfate-polyacrylamide gel electrophoresis and then transferred to polyvinylidene fluoride membranes. After blocking with $5 \%$ nonfat milk for $2 \mathrm{~h}$ at room temperature, the membranes were probed with specific primary antibodies overnight at $4{ }^{\circ} \mathrm{C}$ and then probed with corresponding secondary antibodies for $1 \mathrm{~h}$ at room temperature. Signals were developed using a SuperSignal West Femto Maximum Sensitivity Substrate kit (Thermo). Then, specific protein bands were visualized using the CheniDoc MP Imaging System. Quantification was performed with Image Lab 5.1 (Bio-Rad, Hercules, CA, USA). The detailed antibodies information was listed in Supplementary Table 1.

Nucleus isolation. Nuclear proteins were extracted from AML12 cells using a Nuclear Protein Extract Kit (Beyotime). In brief, $2 \times 10^{6}$ cells were re-suspended in $200 \mu \mathrm{l}$ buffer $\mathrm{A}$ and then vortexed for $5 \mathrm{~s}$. After $15 \mathrm{~min}$ of incubation on ice, $10 \mu \mathrm{l}$ buffer $\mathrm{B}$ was added and then vortexed for $5 \mathrm{~s}$. The supernatant was discarded after centrifugation at $16000 \mathrm{~g}$ for $5 \mathrm{~min}$ at $4^{\circ} \mathrm{C}$. The pellet was re-suspended in $50 \mu \mathrm{l}$ nuclear extraction buffer and then vortexed for $30 \mathrm{~s}$. The nuclei were collected in the supernatant by centrifugation at $16000 \mathrm{~g}$ for $5 \mathrm{~min}$ at $4{ }^{\circ} \mathrm{C}$.

Mitochondrial isolation. Mitochondrial fraction was isolated from cells using mitochondrial isolation buffer ( $\mathrm{pH}: 7.5,210 \mathrm{mM}$ mannitol, $70 \mathrm{mM}$ sucrose, $1 \mathrm{mM}$ EDTA, $10 \mathrm{mM}$ HEPES). In brief, $5 \times 10^{6}$ cells were re-suspended in $1 \mathrm{ml}$ buffer and homogenized for 30-45 seconds on ice using a Dounce Homogenizer at 20000 $\mathrm{rpm} / \mathrm{min}$. Cell slurry was transferred to microfuge tubes and centrifuged at $400 \times \mathrm{g}$ for 10 mins at $4{ }^{\circ} \mathrm{C}$. The supernatant was transferred to a new tube and centrifuged at $10000 \times g$ for $10 \mathrm{~min}$ at $4^{\circ} \mathrm{C}$. The mitochondrial fraction was collected and resuspend in RIPA lysis buffer (Beyotime) containing 1\% protease inhibitor cocktail and $1 \%$ phenylmethane sulfonylfluoride (Sigma).

Mitochondrial membrane potential $(\Delta \Psi \mathrm{m})$ assay. $\Delta \Psi \mathrm{m}$ was determined by the fluorescent dye Rhodamine123 (R123), a cell-permeable cationic dye that preferentially partitions into the mitochondria based on the highly negative $\Delta \Psi \mathrm{m}$. In brief, AML12 cells were treated as described in the cell viability section. Then, the cells were stained with R123 $(10 \mu \mathrm{M})$ for $10 \mathrm{~min}$. Subsequently, the cells were washed twice with PBS, trypsinized, and then collected in a $1.5 \mathrm{ml}$ tube. The change in $\Delta \Psi \mathrm{m}$ was qualitatively observed on an In Cell Analyzer 2000 (GE Healthcare Life Sciences, Chicago, IL, USA).

Immunoprecipitation. Cell lysates $(3 \mathrm{mg}$ protein) were mixed with the indicated antibody $(2 \mu \mathrm{g})$ at $4{ }^{\circ} \mathrm{C}$ overnight. Subsequently, $20 \mu \mathrm{l}$ protein A/G-agarose beads (Santa Cruz Biotechnology, Santa Cruz, CA, USA) were added to the cell lysate and incubated on a rotator for $4 \mathrm{~h}$ at $4{ }^{\circ} \mathrm{C}$. The beads were washed thrice with PBS and then twice with lysis buffer supplemented with complete mini-protease inhibitor cocktail. Bound proteins were boiled in sample preparation buffer for $5 \mathrm{~min}$.

Real-time quantitative PCR. Total DNAs were extracted from AML12 cells with TRIzol (Invitrogen). To determine the ratio of mtDNA/nuclear DNA copy number, a quantitative RT-PCR was performed on Mx3005P qPCR System using SYBR green detecting method (Agilent, Santa Clara, CA, USA). Two pairs of primers were used to quantify and confirm relative mtDNA/nuclear DNA ratio: ND1 for mtDNA, H19 for nuclear DNA. Results were confirmed by at least three independent experiments. All of the PCR primers were synthesized by Sigma. The qPCR program is as follows: $95^{\circ} \mathrm{C} 1 \mathrm{~min}$ for 1 circle; $95^{\circ} \mathrm{C} 15 \mathrm{~s}$, and $60^{\circ} \mathrm{C} 60 \mathrm{~s}$ for
40 cycles. Primers used for the PCR are listed as follows. ND1 forward primer: 5'-AATCGCCATAGCCTTCCTAACAT-3', reverse primer: 5'-GGCGTCTGCAAATGG TTGTAA-3'. H19 forward primer: 5'-GTACCCACCTGTCGTCC-3', reverse primer: 5'-GTCCACGAGACCAATGACTG-3'.

Isolation of primary hepatocytes. Primary hepatocytes were isolated using the two-step Percoll gradient method as previously described. ${ }^{57}$ The liver was perfused with $\mathrm{Ca}^{2+}$ and $\mathrm{Mg}^{2+}$-free Hank's buffered salt solution (HBSS, Gibco, Grand Island, NY, USA) containing EGTA $(2.5 \mathrm{mM})$ and then digested with collagenase buffer $(0.5 \mathrm{mg} / \mathrm{ml}$ collagenase type IV (Roche, Basel, Switzerland), $66.7 \mathrm{mM} \mathrm{NaCl}, 6.7 \mathrm{mM} \mathrm{KCl}, 50 \mathrm{mM}$ HEPES, and $4.8 \mathrm{mM} \mathrm{CaCl}$ ). Digested liver was dissected and then gently teased into small pieces with forceps. Liver slurry was filtered through a $100 \mu \mathrm{m}$ nylon cell strainer (BD). After spin down at $1200 \times \mathrm{g}$ for $5 \mathrm{~min}$, the cell pellet was re-suspended with HBSS. The cell suspension was centrifuged at $400 \times g$ for $3 \mathrm{~min}$, and the pellet was re-suspended in $25 \%$ Percoll and then centrifuged at $550 \times g$ for 5 min with the brake option off. The pellet was washed once with DMEM supplemented with $10 \%$ FBS. Then, the cells were seeded into collagen precoated $100 \mathrm{~mm}$ plates. After $24 \mathrm{~h}$, the adherent cells were collected for the following experiments.

RNA Interference. The shRNA-targeting SIRT3 (mouse, sc-61556) and scrambled RNA (mouse, sc-108060), and shRNA transfection reagent (mouse, sc-108061) were purchased from Santa Cruz Biotechnology. AML12 cells were transfected with $2 \mu \mathrm{g}$ shRNA for $6 \mathrm{~h}$ according to the manufacturer's protocol. Cells were switched to fresh medium and incubated for an additional $24 \mathrm{~h}$. Then, cells were selected with $2 \mu \mathrm{g} / \mathrm{ml}$ puromycin for 6 days, and then $4 \mu \mathrm{g} / \mathrm{ml}$ puromycin for 6 days. Thereafter, cells were pooled together for further experiments.

Statistical analysis. Data were analyzed using GraphPad Prism-6. All experimental data were expressed as mean \pm S.D., and each experiment was performed a minimum of three times. $P<0.05$ was considered to be statistically significant.

\section{Conflict of Interest}

The authors declare no conflict of interest.

Acknowledgements. Financial support by Science and Technology Development Fund, Macao SAR (FDCT 120/2013/A3) and the Research Fund of University of Macau (MYRG2015-00153-ICMS-QRCM and MYRG2017-00109-ICMS-QRCM) are gratefully acknowledged.

\section{Publisher's Note}

Springer Nature remains neutral with regard to jurisdictional claims in published maps and institutional affiliations.

1. Cichoż-Lach $\mathrm{H}$, Michalak A. Oxidative stress as a crucial factor in liver diseases. World $J$ Gastroenterol 2014; 20: 8082-8091.

2. Poelstra K, Schuppan D. Targeted therapy of liver fibrosis/cirrhosis and its complications. $J$ Hepatol 2011; 55: 726-728.

3. Parola M, Robino G. Oxidative stress-related molecules and liver fibrosis. J Hepatol 2001; 35: 297-306.

4. Mantena SK, King AL, Andringa KK, Eccleston HB, Bailey SM. Mitochondrial dysfunction and oxidative stress in the pathogenesis of alcohol- and obesity-induced fatty liver diseases. Free Radic Biol Med 2008; 44: 1259-1272.

5. Watanabe S, Yaginuma R, Ikejima K, Miyazaki A. Liver diseases and metabolic syndrome. J Gastroenterol 2008; 43: 509-518.

6. Nassir F, Ibdah JA. Role of mitochondria in alcoholic liver disease. World J Gastroenterol 2014; 20: 2136-2142.

7. Lombard DB, Alt FW, Cheng HL, Bunkenborg J, Streeper RS, Mostoslavsky R et al. Mammalian Sir2 homolog SIRT3 regulates global mitochondrial lysine acetylation. Mol Cell Biol 2007; 27: 8807-8814.

8. Choudhary C, Kumar C, Gnad F, Nielsen ML, Rehman M, Walther TC et al. Lysine acetylation targets protein complexes and co-regulates major cellular functions. Science 2009; 325: 834-840.

9. Schwer B, North BJ, Frye RA, Ott M, Verdin E. The human silent information regulator (Sir)2 homologue hSIRT3 is a mitochondrial nicotinamide adenine dinucleotide-dependent deacetylase. J Cell Biol 2002; 158: 647-657. 
10. Onyango P, Celic I, McCaffery JM, Boeke JD, Feinberg AP. SIRT3, a human SIR2 homologue, is an NAD-dependent deacetylase localized to mitochondria. Proc Natl Acad Sci USA 2002; 99: 13653-13658.

11. Hallows WC, Yu W, Smith BC, Devries MK, Ellinger JJ, Someya S et al. Sirt3 promotes the urea cycle and fatty acid oxidation during dietary restriction. Mol Cell 2011; 41: 139-149.

12. Palacios OM, Carmona JJ, Michan S, Chen K, Manabe Y, Ward JL 3rd et al. Diet and exercise signals regulate SIRT3 and activate AMPK and PGC-1alpha in skeletal muscle. Aging 2009; 1: 771-783.

13. Lin JD, Handschin C, Spiegelman BM. Metabolic control through the PGC-1 family of transcription coactivators. Cell Metab 2005; 1: 361-370.

14. Tseng AH, Shieh SS, Wang DL. SIRT3 deacetylates FOXO3 to protect mitochondria against oxidative damage. Free Radic Biol Med 2013; 63: 222-234.

15. Qiu X, Brown K, Hirschey MD, Verdin E, Chen D. Calorie restriction reduces oxidative stress by SIRT3-mediated SOD2 activation. Cell Metab 2010; 12: 662-667.

16. Someya S, Yu W, Hallows WC, Xu J, Vann JM, Leeuwenburgh C et al. Sirt3 mediates reduction of oxidative damage and prevention of age-related hearing loss under caloric restriction. Cell 2010; 143: 802-812.

17. Samant SA, Zhang HJ, Hong Z, Pillai VB, Sundaresan NR, Wolfgeher D et al. SIRT3 deacetylates and activates OPA 1 to regulate mitochondrial dynamics during stress. Mol Cell Biol 2014; 34: 807-819.

18. Subramanian C, Jarzembowski JA, Opipari AW Jr., Castle VP, Kwok RP. HDAC6 deacetylates Ku70 and regulates Ku70-Bax binding in neuroblastoma. Neoplasia 2011; 13: 726-734.

19. Sundaresan NR, Samant SA, Pillai VB, Rajamohan SB, Gupta MP. SIRT3 is a stressresponsive deacetylase in cardiomyocytes that protects cells from stress-mediated cell death by deacetylation of Ku70. Mol Cell Biol 2008; 28: 6384-6401.

20. Morio LA, Chiu H, Sprowles KA, Zhou P, Heck DE, Gordon MK et al. Distinct roles of tumor necrosis factor-alpha and nitric oxide in acute liver injury induced by carbon tetrachloride in mice. Toxicol Appl Pharmacol 2001; 172: 44-51.

21. Yang YJ, Hubbard BP, Sinclair DA, Tong Q. Characterization of murine SIRT3 transcript variants and corresponding protein products. J Cell Biochem 2010; 111: 1051-1058.

22. Halliwell B. Biochemistry of oxidative stress. Biochem Soc Trans 2007; 35: 1147-1150

23. Pompella A, Visvikis A, Paolicchi A, De Tata V, Casini AF. The changing faces of glutathione, a cellular protagonist. Biochem Pharmacol 2003; 66: 1499-1503.

24. Gorrini C, Harris IS, Mak TW. Modulation of oxidative stress as an anticancer strategy. Nat Rev Drug Discov 2013; 12: 931-947.

25. Nguyen T, Sherratt PJ, Nioi P, Yang CS, Pickett CB. Nrf2 controls constitutive and inducible expression of ARE-driven genes through a dynamic pathway involving nucleocytoplasmic shuttling by Keap1. J Biol Chem 2005; 280: 32485-32492.

26. Whitaker RM, Corum D, Beeson CC, Schnellmann RG. Mitochondrial biogenesis as a pharmacological target: a new approach to acute and chronic diseases. Annu Rev Pharmacol Toxicol 2016; 56: 229-249.

27. Kong XX, Wang R, Xue Y, Liu XJ, Zhang HB, Chen $Y$ et al. Sirtuin 3, a new target of PGC-1alpha, plays an important role in the suppression of ROS and mitochondrial biogenesis. PLOS ONE 2010; 5: e11707.

28. Cheng Y, Ren X, Gowda AS, Shan Y, Zhang L, Yuan YS et al. Interaction of Sirt3 with OGG1 contributes to repair of mitochondrial DNA and protects from apoptotic cell death under oxidative stress. Cell Death Dis 2013; 4: e731.

29. Zamzami N, Marchetti P, Castedo M, Decaudin D, Macho A, Hirsch T et al. Sequentia reduction of mitochondrial transmembrane potential and generation of reactive oxygen species in early programmed cell death. J Exp Med 1995; 182: 367-377.

30. Smirnova E, Griparic L, Shurland DL, van der Bliek AM. Dynamin-related protein Drp1 is required for mitochondrial division in mammalian cells. Mol Biol Cell 2001; 12: 2245-2256.

31. Loson OC, Song Z, Chen H, Chan DC. Fis1, Mff, MiD49, and MiD51 mediate Drp1 recruitment in mitochondrial fission. Mol Biol Cell 2013; 24: 659-667.

32. Karbowski M, Lee YJ, Gaume B, Jeong SY, Frank S, Nechushtan A et al. Spatial and temporal association of Bax with mitochondrial fission sites, Drp1, and Mfn2 during apoptosis. J Cell Biol 2002; 159: 931-938.

33. Wu SG, Zhou FF, Zhang ZZ, Xing D. Bax is essential for Drp1-mediated mitochondrial fission but not for mitochondrial outer membrane permeabilization caused by photodynamic therapy. J Cell Physiol 2011; 226: 530-541.

34. Kendrick AA, Choudhury M, Rahman SM, McCurdy CE, Friederich M, Van Hove JL et al. Fatty liver is associated with reduced SIRT3 activity and mitochondrial protein hyperacetylation. Biochem J 2011; 433: 505-514.

35. Ren JH, Chen X, Zhou L, Tao NN, Zhou HZ, Liu B et al. Protective role of sirtuin3 (SIRT3) in oxidative stress mediated by hepatitis B virus X protein expression. PLoS One 2016; 11 e0150961.

36. Jaeschke $\mathrm{H}$, Ramachandran $\mathrm{A}$. Reactive oxygen species in the normal and acutely injured liver. J Hepatol 2011; 55: 227-228.

37. Tao R, Coleman MC, Pennington JD, Ozden O, Park SH, Jiang $\mathrm{H}$ et al. Sirt3-mediated deacetylation of evolutionarily conserved lysine 122 regulates MnSOD activity in response to stress. Mol Cell 2010; 40: 893-904.
38. Hayes JD, McLellan LI. Glutathione and glutathione-dependent enzymes represent a coordinately regulated defence against oxidative stress. Free Radic Res 1999; 31: 273-300.

39. Giudice A, Arra C, Turco MC. Review of molecular mechanisms involved in the activation of the Nrf2-ARE signaling pathway by chemopreventive agents. Methods Mol Biol 2010; 647 $37-74$.

40. Satterstrom FK, Swindell WR, Laurent G, Vyas S, Bulyk ML, Haigis MC. Nuclear respiratory factor 2 induces SIRT3 expression. Aging Cell 2015; 14: 818-825.

41. Brenmoehl J, Hoeflich A. Dual control of mitochondrial biogenesis by sirtuin 1 and sirtuin 3 . Mitochondrion 2013; 13: 755-761.

42. Zhao H, Ding T, Ren Y, Li T, Li R, Fan Y et al. Role of Sirt3 in mitochondrial biogenesis and developmental competence of human in vitro matured oocytes. Hum Reprod 2016; 31 607-622.

43. Hawley SA, Davison M, Woods A, Davies SP, Beri RK, Carling D et al. Characterization of the AMP-activated protein kinase kinase from rat liver and identification of threonine 172 as the major site at which it phosphorylates AMP-activated protein kinase. J Biol Chem 1996; 271: 27879-27887.

44. Wan Z, Root-McCaig J, Castellani L, Kemp BE, Steinberg GR, Wright DC. Evidence for the role of AMPK in regulating PGC-1 alpha expression and mitochondrial proteins in mouse epididymal adipose tissue. Obesity 2014; 22: 730-738.

45. Jager S, Handschin C, St-Pierre J, Spiegelman BM. AMP-activated protein kinase (AMPK) action in skeletal muscle via direct phosphorylation of PGC-1alpha. Proc Natl Acad Sci USA 2007; 104: 12017-12022.

46. Shi T, Fan GQ, Xiao SD. SIRT3 reduces lipid accumulation via AMPK activation in human hepatic cells. J Dig Dis 2010; 11: 55-62.

47. Joo MS, Kim WD, Lee KY, Kim JH, Koo JH, Kim SG. AMPK facilitates nuclear accumulation of Nrf2 by phosphorylating at serine 550. Mol Cell Biol 2016; 36: 1931-1942.

48. Smith RA, Hartley RC, Cocheme HM, Murphy MP. Mitochondrial pharmacology. Trends Pharmacol Sci 2012; 33: 341-352

49. Tchou J, Grollman AP. Repair of DNA containing the oxidatively-damaged base 8-oxoguanine. Mutat Res 1993; 299: 277-287.

50. de Souza-Pinto NC, Eide L, Hogue BA, Thybo T, Stevnsner T, Seeberg E et al. Repair of 8-oxodeoxyguanosine lesions in mitochondrial dna depends on the oxoguanine dna glycosylase (OGG1) gene and 8-oxoguanine accumulates in the mitochondrial dna of OGG1-defective mice. Cancer Res 2001; 61: 5378-5381.

51. Hirschey MD, Shimazu T, Jing E, Grueter CA, Collins AM, Aouizerat B et al. SIRT3 deficiency and mitochondrial protein hyperacetylation accelerate the development of the metabolic syndrome. Mol Cell 2011; 44: 177-190.

52. Smirnova E, Shurland DL, Ryazantsev SN, van der Bliek AM. A human dynamin-related protein controls the distribution of mitochondria. J Cell Biol 1998; 143: 351-358.

53. Szabadkai G, Simoni AM, Chami M, Wieckowski MR, Youle RJ, Rizzuto R. Drp-1-dependent division of the mitochondrial network blocks intraorganellar $\mathrm{Ca}^{2+}$ waves and protects against $\mathrm{Ca}^{2+}$-mediated apoptosis. Mol Cell 2004; 16: 59-68.

54. Yang YJ, Cimen H, Han MJ, Shi T, Deng JH, Koc H et al. NAD+-dependent deacetylase SIRT3 regulates mitochondrial protein synthesis by deacetylation of the ribosomal protein MRPL10. J Biol Chem 2010; 285: 7417-7429

55. Lin LG, Pan WJ, Chen KY, Wang F, Gengler J, Sun YX et al. Adipocyte expression of PU.1 transcription factor causes insulin resistance through upregulation of inflammatory cytokine gene expression and ROS production. Am J Physiol Endocrinol Metab 2012; 302 E1550-E1559.

56. Wang $A Q$, Wang SP, Jiang $Y$, Chen MW, Wang YT, Lin LG. Bio-assay guided identification of hepatoprotective polyphenols from Penthorum chinense Pursh on $t$-BHP induced oxidative stress injured L02 cells. Food Funct 2016; 7: 2074-2083.

57. Kim HJ, Joe Y, Yu JK, Chen YQ, Jeong SO, Mani N et al. Carbon monoxide protects against hepatic ischemia/reperfusion injury by modulating the miR-34a/SIRT1 pathway. Biochim Biophys Acta 2015; 1852: 1550-1559.

Cell Death and Disease is an open-access journal published by Nature Publishing Group. This work is licensed under a Creative Commons Attribution 4.0 International License. The images or other third party material in this article are included in the article's Creative Commons license, unless indicated otherwise in the credit line; if the material is not included under the Creative Commons license, users will need to obtain permission from the license holder to reproduce the material. To view a copy of this license, visit http://creativecommons.org/licenses/by/4.0/

(C) The Author(s) 2017 